\title{
Erratum
}

J. Microbiol. Biotechnol. (2018), 28(11), 1800-1805

https://doi.org/10.4014/jmb.1805.05072

\section{Erratum to: Orally-Administered Bifidobacterium animalis subsp. lactis Strain BB12 on Dextran Sodium Sulfate-Induced Colitis in Mice} Jung Min Chae ${ }^{1}$, Wan Heo ${ }^{1}$, Hyung Taek Cho ${ }^{1}$, Dong Hun Lee ${ }^{1}$, Jun Ho Kim ${ }^{2}$, Min Suk Rhee ${ }^{3}$, Tae-Sik Park ${ }^{4}$, Yong Ki Kim ${ }^{5}$, Jin Hyup Lee ${ }^{1}$, and Young Jun Kim ${ }^{1 *}$

${ }^{1}$ Department of Food and Biotechnology, Korea University, Sejong 30019, Republic of Korea

${ }^{2}$ Department of Food Science and Biotechnology, Andong National University, Andong 36729, Republic of Korea

${ }^{3}$ Department of Biotechnology, Korea University, Seoul 02841, Republic of Korea

${ }^{4}$ Department of Life Science, Gachon University, Seongnam 13120, Republic of Korea

${ }^{5}$ Maeil Dairies Co., Ltd RED Center, Pyeongtaek 7714, Republic of Korea

This erratum is being published to correct the author's contribution of above manuscript by Chae et al. that was published in Journal of Microbiology and Biotechnology (2018, 28:11, 1800-1805).

The ninth author (Jin Hyup Lee) should be marked as corresponding author ${ }^{*}$ ) with latest author (Young Jun Kim).

The correspondence should appear as:

${ }^{*}$ Corresponding authors

Young Jun Kim

Phone: +82-44-860-1435;

Fax: +82-44-860-1586;

E-mail: yk46@korea.ac.kr

Jin Hyup Lee

Phone: $+82-44-860-1437$

Fax: +82-44-860-1586;

E-mail: jinhyuplee@korea.ac.kr 